\title{
Study on Low-carbon Urban Development Path and Strategy Based on Principal Components Analysis
}

\author{
Wang Hongping*, Su Tianbao and Sun Hailing
}

Henan University of Urban Construction, Pingdingshan, Henan, 467036, China

\begin{abstract}
Scholars at home and abroad always focus on the low-carbon urban development. This paper described the basic theory of low-carbon city, analyzed the main factors effect the urban carbon emissions based on principal components analysis method, and established evaluation model to adapt to the development of low-carbon city. Finally, reasonable proposals have been proposed to provide scientific and theoretical basis for government decision making.
\end{abstract}

Keywords: Evaluation model, low-carbon urban, principal components analysis.

\section{INTRODUCTION}

Decades after reform and opening up policy implementation, China's economy has been developed rapidly. In the process of the development, large-medium cities were leaders in national economic development who played leading role on economic development. However, the consumption of resources has been intensified, correspondingly leading the growth rate of total carbon emissions far more than the speed of the economic development [1].

Statistics show that the carbon emissions have been increasing annually in China around the world. The total amount of carbon emissions in China has reached to $26.72 \%$ of the world until 2012, far more than many developed countries, $86 \%$ of which are concentrated in cities. Throughout decades of China's economic development, series of problems emerged prominently owing to increasing urban carbon emissions, such as urban heat island effect, energy efficiency and air problems, causing high pressure of carbon emissions reduction in our country. If this high consumption, high emissions, and high-growth urban development model does not change, China will face the great risk of "carbon lock-in" and pay a much higher abatement cost. Thus, the identification of the key factors affect low-carbon urban development will contribute to find the key points to promote the development of low carbon city. However, the current research of low-carbon urban development is unbalanced. Much attention has been paid in qualitative terms, while the quantitative study of the factors which impact on the development of the low-carbon urban has been neglected. But, there's no sophisticated evaluation system in study of the low-carbon urban development. The existed evaluation system is not fully based on the theory of low-carbon urban development and can not completely reflect the needs of low-carbon urban construction, too.

*Address correspondence to this author at the Henan University of Urban Construction, Pingdingshan, Henan, 467036, China; Tel: 15003757299;

E-mail: 396753200@qq.com
In summary, this essay introduced basic theory of lowcarbon urban firstly, and then analyzed the factors influenced carbon emissions based on the basis of principal component analysis method. Finally, reasonable proposals have been proposed to provide scientific and theoretical basis for government decision making [2].

\section{PRINCIPAL COMPONENTS ANALYSIS}

\subsection{Fundamental of Principal Component Analysis}

As a kind of statistical analysis method, principal component analysis always uses comprehensive indexes to summarize multiple indexes. The basic principle is to collect original data information from a large amount of observation data to explain the original data, so as to simplify the structure of data and reduce the dimension of multivariate, make the research and analysis of the problems convenient [3].

\subsection{Specific Steps}

(1) The p-dimensional random vector of standardization sample data by original statistical collection constitutes the samples of

$$
\begin{aligned}
& X=\left(\begin{array}{llll}
X_{1} & X_{2} & \cdots & X_{p}
\end{array}\right)^{T} \\
& \text { of which } x_{i}=\left(\begin{array}{llll}
x_{1} & x_{2} & \cdots & x_{p}
\end{array}\right)^{T} \mathrm{i}=1,2 \ldots \mathrm{n}, \mathrm{n}>\mathrm{p} .
\end{aligned}
$$

Built sample matrix for the original random vector and standardize it:

$Z_{i j}$-The corresponding standardized variable of original sample $X_{i j}$

$Z_{i j}=\frac{x_{i j}-\bar{x}_{j}}{s_{j}}(i=1,2 \ldots n ; j=1,2 \ldots p)$

$X_{j}$-The corresponding average variable of original sample $X_{i j}$

$X_{j}=\frac{\sum_{i=1}^{n} X_{i j}}{n}$ 
$S_{j}$-The corresponding standard deviation variable of original sample $X_{i j}$

$S_{j}=\sqrt{\frac{\sum_{i=1}^{n}\left(X_{i j}-\bar{X}_{j}\right)}{n-1}}$

(2) Calculate correlation Evaluation coefficient matrix $R$ standardized, of which $Z_{j}=\left(\begin{array}{lllll}Z_{1 j} & Z_{2 j} & \cdots & Z_{3 j}\end{array}\right)$ is R. Using $r_{j i}$ to denote the correlation coefficient between $x_{j}$ and $x_{i}$, we can establish matrix

$$
\begin{aligned}
& R=\left|\begin{array}{cccc}
r_{11} & r_{12} & \cdots & r_{1 p} \\
r_{21} & r_{22} & \cdots & r_{2 p} \\
\cdots & \cdots & \cdots & \cdots \\
r_{p 1} & r_{p 2} & \cdots & r_{p p}
\end{array}\right| \cdot \\
& r_{j i}=\frac{\sum\left(X_{k 1}-\bar{X}_{j}\right)}{\sqrt{\sum_{k=1}^{n}\left(X_{k 1}-\bar{X}_{i}\right) \sqrt{\sum_{k=1}^{n}\left(X_{k 1}-\bar{X}_{j}\right)}}}(i, j=1,2 \ldots p)
\end{aligned}
$$

(3) Then, calculate eigenvectors and eigenvalues of matrix $\mathrm{R}$, ascertain the principal components and the number of the factors. P relevant eigenvalues could be obtained by solving the characteristic equation of $\left|R-\lambda I_{P}\right|=0$. Determine the value $\mathrm{m}$ of principal component factors on the basis of

$$
\frac{\sum_{j=1}^{m} \lambda_{j}}{\sum_{j=1}^{p} \lambda_{j}} \geq 0.85
$$

of which the corresponding eigenvector to $\lambda_{j}(j=1,2 \ldots, m$,$) is$ $a_{k}=\left(\begin{array}{llll}a_{k 1} & a_{k 2} & \cdots & a_{k m}\end{array}\right)$.

On the above analysis, we can take the main ingredient corresponding to $\lambda_{j}$ as

$a_{k}=\frac{p_{j}}{\sqrt{\lambda_{k}}}$,

- the $\mathrm{j}$ eigenvalue of matrix $\mathrm{R}, p_{j}$ - constructed which $\mathrm{j}$ principal component. $p_{j}$ - the composite vector constructed by standardized variables $Z_{1}, Z_{2} \cdots Z_{p}$ in $\mathrm{j}$-th principal component [4].

\section{MAIN FACTORS AFFECT THE ANALYSIS OF LOW-CARBON URBAN DEVELOPMENT}

There are many factors affecting low-carbon urban development, such as economic development index, social development index, low-carbon development index etc. This essay analyzes the factors that affect low-carbon development index from the perspective of qualitative, including per capita building carbon emissions, elasticity coefficient of energy consumption, energy consumption of unit of GDP, the proportion of tertiary industry in GDP, green coverage, the efficiency of public transport, the compliance rate of industrial wastewater discharge. The factors of economic and society has not been considered in this paper due to its small influence on the low-carbon urban development [5].

\section{(1) Per Capita building Carbon Emissions}

Statistics show building carbon emissions accounted for $30 \%$ of the total greenhouse gas emissions around the world. Building carbon emissions refers to the primary energy consumption in the whole life cycle of the building. Throughout the entire life cycle, Building carbon emissions is an important factor affecting the urban low carbonization. In general, it's a negative correlation between per capita building carbon emissions and the low-carbon degree of the city.

\section{(2) Elasticity coefficient of Energy Consumption}

Energy consumption elasticity coefficient is an indicator reflecting the relationship between the growth rate of energy consumption and economic, which is equal to the ratio of average annual growth rate of energy consumption and the corresponding GDP over the same period. It means that how much the growth degree of the energy consumption caused by certain the growth of the GDP. The carbon emissions mainly produced in the process of industrial production, which has a significant impact on carbon emissions. Therefore, the elasticity of energy consumption is another important factor affecting the low-carbon urban process, which presents a negative correlation with the process of lowcarbon urban.

\section{(3) Energy Consumption Per Unit of GDP}

Energy consumption of unit of GDP refers to the energy required for the increase of $10,000 ¥$ of the GNP in a certain period. It can reflect the utilization efficiency of energy directly. The higher the total unit is, the lower the energy utilization efficiency will be. It is also one of the important indicators to measure the effect of low-carbon urban development, presents a negative correlation with the process of low-carbon urban.

\section{(4) The Proportion of Tertiary Industry in GDP}

Tertiary Industry, a typical low-carbon industry, consumes less fossil energy. The proportion of tertiary industry in GDP indicates the structure and the level of a region's economic. The higher the proportion of tertiary industry is, the better the region's economic structure and the lower carbon emissions will be. Therefore, the proportion of tertiary industry in GDP has a positive relationship with the lowcarbon urban.

\section{(5) Green Coverage}

Green coverage is a ratio between the urban green area with the urban land area. Green coverage $(\%)=$ total area of urban green space / total area of urban land * $100 \%$. It's a main indicator measuring the level of an urban greening and the degree of low-carbon city. The higher the green coverage means a higher level of low-carbon urban. Therefore, the green coverage rate and the level of low-carbon urban appears a positive correlation relationship.

\section{(6) The Efficiency of Public Transport}

The efficiency of public transport refers to the average amount of passengers for each public transport every year. Studies show that there is a certain substitution effect for private cars with bus, urban rail transit. We can reduce the number of private car use by perfecting public transit and improving the operational efficiency of public transport sys- 
tems, and thus reduce carbon dioxide emissions. Therefore, the efficiency of public transport operational has a positive relationship with the urban low carbonization.

(7) The Compliance Rate of Industrial Wastewater Discharge

The compliance rate of industrial wastewater discharge refers to the amount of urban industrial wastewater discharge compliance accounts for the percentage of total industrial wastewater discharge. It's close to the level of low-carbon city. The higher the urban industrial wastewater discharge compliance rate is, the lower the carbon emissions will be. Therefore, the industrial wastewater discharge compliance rate and the level of low-carbon urban exhibits a positive correlation [6-9].

\section{THE EVALUATION MODEL OF LOW-CARBON URBAN DEVELOPMENT BASED ON PRINCIPAL COMPONENT ANALYSIS}

The evaluation model of low carbon city development has been established on ascertaining the weights of evaluation factors by principal component analysis:

$Y_{k}^{1}=\sum_{j=1}^{n} X_{j} C_{j}$

$Y_{k}^{1}$ - index value of item 1 in the year $\mathrm{K}, C_{j}$ - principal component scores of the comprehensive factors, n-the number of principal components, $X_{j}$ - the weight of each comprehensive factor (the contribution rate of principal components)

Select the statistical data of Henan Province. Supposing: $X_{1}$ - Carbon emissions per capita construction; $X_{2}$ - Elasticity of energy consumption; $X_{3}$ - Energy consumption per unit of GDP; $X_{4}$ - The proportion of tertiary industry in GDP; $X_{5}$ - The green coverage rate; $X_{6}$ - Operational efficiency of public transport; $X_{7}$ - Industrial wastewater discharge compliance rate. Through the logistics and dimensionless transformation, the low-carbon development of Henan Province in the last 10 years can be obtained. As shown in Table $\mathbf{1}$.
According to the calculation method of eigenvectors and eigenvalues, eigenvectors, contribution rate and cumulative contribution rate of $\mathrm{R}$ can be calculated. Then, the principal component analysis and selection can be got. As shown in Table 2.

According to Table 2, three eigenvalues $>1$ and the cumulative of variance has reached to $94.134 \%$, which indicates that the principal components selected can reach the basic requirements and explain the system sufficiently. So we can choose three main components and calculate the score of principal component by SPSS system. As shown in Table 3.

As shown in principal component 1 from Table 3 , the coefficient of absolute value $X_{2}, X_{4}, X_{5}$ were around 0.3 which indicates the importance of changing the proportion of industry and improving the energy utilization to low-carbon urban development. In principal component 2 , the coefficient of absolute value $X_{1}, X_{3}$ were about 0.5 which shows the importance of energy conservation and emission reduction to low-carbon development. In principal component 3, the coefficient of absolute value $X_{6}, X_{7}$ were about 0.5 which shows that the promotion of public transportation and the improvement of the industrial wastewater treatment are positive to the low-carbon development, too. Based on the above analysis, the formulation can be obtained as follows:

$F_{1}=-0.1 X_{1}+0.297 X_{2}+0.016 X_{3}+0.737 X_{4}$

$+0.368 X_{5}+0.067 X_{6}+0.102 X_{7}$

$F_{1}=-0.498 X_{1}+0.106 X_{2}+0.487 X_{3}-0.053 X_{4}-0.095 X_{5}$ -

$0.154 X_{6}-0.025 X_{7}$

$F_{1}=-0.026 X_{1}+0.062 X_{2}-0.117 X_{3}-0.177 X_{4}$

$+0.08 X_{5}+0.539 X_{6}-0.491 X_{7}$

Similarly, we select the contribution rate of cumulative variance as weights of composite score, getting the expression of the level of development of low-carbon composite score:

Table 1. Low-carbon development level in Henan Province during 2001-2010.

\begin{tabular}{|c|c|c|c|c|c|c|c|}
\hline 2001 & 2210.67 & 1.1 & 0.18 & 36 & 0.32 & 14 & 84.79 \\
\hline 2003 & 1342.22 & 0.89 & 0.14 & 40 & 0.45 & 8.85 & 96.33 \\
\hline 2004 & 721.68 & 0.98 & 0.09 & 40 & 0.41 & 9.91 & 97.2 \\
\hline 2006 & 418.52 & 1.15 & 0.01 & 40 & 0.53 & 11.71 & 96.65 \\
\hline 2007 & 911.99 & 1.41 & 0.05 & 40 & 0.56 & 13.08 & 86.38 \\
\hline 2008 & 1450.46 & 1.49 & 0.12 & 41 & 0.61 & 11.34 & 86.09 \\
\hline 2009 & 1650.35 & 2.5 & 0.19 & 41 & 0.67 & 13.24 & 90.83 \\
\hline
\end{tabular}


Table 2. The eigenvectors, the contribution rate and the cumulative contribution rate of $R$.

\begin{tabular}{|c|c|c|c|}
\hline Principal Component & The eigenvectors & The contribution rate & The cumulative contribution rate \\
\hline \hline 1 & 3.103 & 44.327 & 44.327 \\
\hline 2 & 2.052 & 29.321 & 73.648 \\
\hline 3 & 1.434 & 20.486 & 94.134 \\
\hline 4 & 0.327 & 4.669 & 98.803 \\
\hline 5 & 0.041 & 0.587 & 99.39 \\
\hline 6 & 0.029 & 0.409 & 99.799 \\
\hline 7 & 0.014 & 0.201 & 100 \\
\hline
\end{tabular}

Table 3. Principal component score coefficient.

\begin{tabular}{|c|c|c|c|c|c|c|c|}
\hline Index & $X_{1}$ & $X_{2}$ & $X_{3}$ & $X_{4}$ & & $X_{6}$ & $X_{7}$ \\
\hline Principal component 1 & -0.1 & 0.297 & 0.016 & 0.373 & 0.368 & 0.067 & 0.102 \\
\hline Principal component 2 & 0.498 & 0.106 & 0.487 & -0.053 & -0.095 & -0.154 & -0.025 \\
\hline Principal component 3 & -0.026 & 0.062 & -0.117 & -0.177 & 0.08 & 0.539 & -0.491 \\
\hline
\end{tabular}

Table 4. The main component score and the composite score of the level of development of low-carbon.

\begin{tabular}{|c|c|c|c|c|c|c|c|c|c|c|}
\hline & 2001 & 2002 & 2003 & 2004 & 2005 & 2006 & 2007 & 2008 & 2009 & 2010 \\
\hline Principal Component 1 & -1.699 & -0.873 & -0.404 & -0.349 & -0.287 & 0.135 & 0.107 & 0.368 & 1.108 & 1.895 \\
\hline Principal Component 2 & 1.131 & 1.022 & 0.310 & -0.525 & -1.406 & -1.489 & -0.826 & 0.124 & 0.692 & 0.966 \\
\hline Principal Component 3 & 1.574 & -1.497 & -1.132 & -0.849 & -0.174 & -0.092 & 1.207 & 0.582 & 0.623 & -0.241 \\
\hline Composite Score & -0.099 & -0.394 & -0.320 & -0.483 & -0.575 & -0.395 & 0.053 & 0.319 & 0.821 & 1.073 \\
\hline
\end{tabular}

Table 5. Types of low-carbon economy.

\begin{tabular}{|c|c|}
\hline Composite Index & Type of Economy \\
\hline \hline Less than 0.42 & Ultrahigh-carbon economy \\
\hline $0.42 \sim 0.675$ & High-carbon economy \\
\hline $0.675 \sim 0.875$ & The higher carbon economy \\
\hline $0.875 \sim 1$ & Medium-carbon economy \\
\hline $1.0 \sim 1.165$ & Low-carbon economy \\
\hline More than 1.165 & Lower-carbon economy \\
\hline
\end{tabular}

$$
\begin{gathered}
F=\frac{\lambda_{5}}{\sum_{i=5}^{11} \lambda_{j}} F_{1}+\frac{\lambda_{6}}{\sum_{i=5}^{11} \lambda_{j}} F_{2}+\frac{\lambda_{7}}{\sum_{i=5}^{11} \lambda_{j}} F_{3} \\
=0.443 F_{1}+0.293 F_{1}+0.205 F_{1}
\end{gathered}
$$

Then, we can to calculate the scores of principal component and composite score of low-carbon development by using SPSS software as shown in Table 4, and finally got the tendency chart as shown in Fig. (1).

Comparing the composite score of the main component of low-carbon level with the evaluation standard, the level of low-carbon economic development of the city could be judged as shown in Table $\mathbf{5}$. 


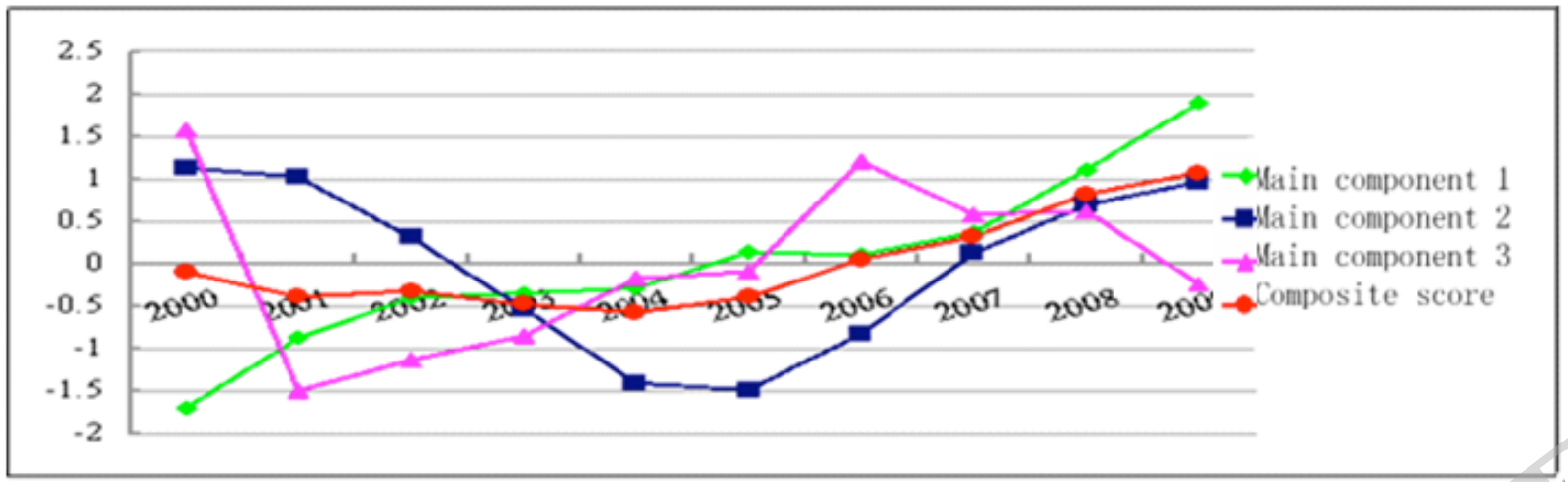

Fig. (1). The principal component and composite score of low-carbon development.

\section{CONCLUSION}

According to Fig. (1), principal component 1-Carbon emissions per capita construction is in a decline trend; principal component 2-the curve of elasticity of energy consumption presented a fluctuation, which showed a remarkable decline trend since 2001 and reached the lowest in 2005. Then, it began to increase gradually, which probably caused by the increasing of infrastructure investment with a higher per capita CO2 emissions; principal component 3-energy consumption of unit GDP showed a downward trend since 2006. As for Henan Province, the low-carbon construction increase steadily in the last 10 years, but it had a remarkable increase since 2006. It also shows that positive and effective measures such as improving energy efficiency, adjusting the industrial structure and green construction have been taken in Henan Province, which made the low-carbon urban construction continuously developed.

However, comparing the level of china's low carbon evaluation standard, it is still in the early stage of low-carbon urban construction in Henan. There are also many problems. First, it has not yet built a reasonable path of low-carbon urban construction on the economic development. Second, it requires long-term efforts to change the urban energy structure which mainly depend on coal as the main energy. Third, it is still not clear about the main adjustment path of the industrial structure on low-carbon economy. Fourth, it still hasn't uniform design standards for building carbon reduction. Last, people need to raise their awareness of lowcarbon.

To overcome these problems, it is proposed to combine the development of low-carbon economy with the construction of low-carbon city, take the three principal components factors as the key points, and make build low-carbon cities as our strategic objectives in the future, finally to realize the China's sustainable development. So the keynotes of future of low-carbon urban development should focus on the following aspects:

First, strengthen propagandize to improve people's consciousness of low-carbon and public participation, strictly control carbon emissions of building and traffic in people's daily life, and establish a advantage group of low-carbon in the community.
Second, raise the proportion of secondary industry and tertiary industry to reduce carbon emissions by lessening GDP energy consumption.

Third, compulsive measures should be established by government departments. For example, we can take lowcarbon development index as a rigid index to reduce per capita building carbon emissions. At the same time, establish the corresponding incentive mechanism to implement preferential tax policy for the low-carbon project. Otherwise, we can introduce in solar technology, geothermal, hydropower, biomass, wind and other new energy and renewable energy during the architectural planning and design, too.

Shortly, low-carbon city construction is a long-term and complicated systematic engineering. It can be seen that China's low-carbon urban development is still in preliminary stage comparing with developed countries by using principal component analysis in the evaluation model of low-carbon urban development. Therefore, it has great practical significance to identify the main factors affecting the development of low-carbon city, and establish a scientific, rational and effective low-carbon city evaluation system to measure the degree of compliance with the city's sustainable development.

\section{CONFLICT OF INTEREST}

The authors confirm that this article content has no conflict of interest.

\section{ACKNOWLEDGEMENTS}

The article is part of the achievement of the Natural Science Project "Research on ecological planning of the small town in Henan Province". Project number: 20111282.

\section{REFERENCES}

[1] K. Xia, "Promote the low-carbon economy development to realize city's sustainable development," Environmental Protection, no.7, 2008.

[2] B. An, "Low-carbon urban: a new bright spot of urban development," Environmental protection and circular economy,

[3] D. Yu, "World Meteorological Organization: the concentration of greenhouse gases is at a high level," Caijing.com, pp. 11.27, 2008.

[4] S. Jin, "WWF Launching China's low-carbon urban development projects," Environmental Protection, no. 2, 2008. 
[5] Sustainable Development Research Group of Chinese Academy Science. China's sustainable development strategy report for 2009[M], Beijing: Science Press, 2009.

[6] Y. Fu, Y. Wang, and D. Li, "Research on the development path of low-carbon urban," Impact of Science On Society, no. 2, pp. 5-10, 2008.

[7] L. G. Edward, and E. K. Matthew, "The greenness of cities: Carbon dioxide emissions and urban development," Journal of Urban Economics, vol. 67, pp. 404-418, 2010.
[8] Emissions and urban development," Journal of Urban Economics, vol. 67 , pp. 404-418, 2010.

[9] G. Cheirs, "How to live a low-carbonlive: the individual's guide to stopping climate chmge," London Sterling, 2007.

Received: April 10,2015

(c) Hongping et al.; Licensee Bentham Open.

This is an open access article licensed under the terms of the (https://creativecommons.org/licenses/by/4.0/legalcode), which permits unrestricted, noncommercial use, distribution and reproduction in any medium, provided the work is properly cited. 